\title{
Journal of Dentistry and Oral Care
}

\section{Unlocking the Student's Potential: Training Students to Fabricate Tooth Colored Restorations Utilizing CAD/CAM Technology in Two Progressive Modes}

\author{
Maria P. Rodriguez Cardenas ${ }^{1 *}$, Angela DeBartolo ${ }^{2}$, Mark S. Wolff ${ }^{3}$
}

${ }^{1}$ Department of Cariology and Comprehensive Care ${ }^{2}$ Department of Cariology and Comprehensive Care ${ }^{3}$ Department of Cariology and Comprehensive Care

\section{Received date: April 21, 2015 \\ Accepted date: May 8, 2015 \\ Published date: May 14, 2015}

*Corresponding Author: Maria P. Rodriguez Cardenas, Department of Cariology and Comprehensive Care New York University College of Dentistry (NYUCD), New York, USA.E-mail: mpr217@nyu.edu

Citation: : Rodriguez Cardenas, M. P., et al. Unlocking the Student's Potential: Training Students to Fabricate Tooth Colored Restorations Utilizing CAD/CAM Technology in Two Progressive Modes. (2015) J Dent \& Oral Care 1(1): 35-37.

Objective: The objective of this paper is to explain two of the different modalities that the CEREC CAD/CAM system offers, the Biogeneric Copy and Biogeneric Individual utilizing digital impression to design and fabricate chair side $\mathrm{CAD} / \mathrm{CAM}$ restorations, that are taught to students at New York University College of Dentistry (NYUCD).

\section{Introduction}

Computer Aided Design and Computer Aided Manufacture, also known as CAD/CAM, is one of the fastest growing segments of dentistry ${ }^{[1-4]}$. Dental Professionals can now utilize new and affordable technologies to be guided in the placement of implants as well as the design and fabrication of implant abutments, dentures, crown, bridges, and tooth colored inlays and onlays. These restorative procedures, which traditionally required multiple patient and laboratory visits to complete, can frequently be completed either chair side (direct) in a single visit, or laboratory manufactured (indirect) in multiple visits ${ }^{[5-7]}$. The advantages of fabricating the restoration chair side are multiple; patients require only a single visit and local anesthesia experience, there is no need for an uncomfortable (often messy) elastomeric impression in the patient's mouth, there is no need to fabricate a temporary restoration and there is no laboratory procedure requiring the patient to return for an additional office visit for insertion.

Laboratory manufactured restorations utilize digital images, generated chair side by the dentist, that are electronically (and securely) forwarded to the dental laboratory. The dental technicians utilize the digital image to create virtual models that allow them to virtually fabricate the restoration using software to design and modify the restoration optimizing the fit, contour and occlusion of the virtual final restoration. When the virtual restoration meets all quality assurance steps (such as adequate contact, contour and occlusal thickness) the final restoration is sent to the computerized milling unit for fabrication. After finishing and polishing the restoration, it is returned to the dentist for try in and final insertion. As computer technology continues to grow and improve, advances in the field dental CAD/CAM will be more cost-effective, automated to utilize and precise in fit and anatomic form ${ }^{[1-6]}$ Within the academic setting, CAD/CAM technology can be utilized to better educate students by improving their skills in assessing their preparation ${ }^{[2-4]}$.

Copyrights: (C) 2015 Rodriguez Cardenas, M. P. This is an Open access article distributed under the terms of Creative Commons 
At NYUCD, as part of the esthetic dentistry curriculum taught to all students, the students are trained by certified faculty, in the pre-clinic environment to provide the foundational knowledge and mechanical skills to utilize CAD/CAM technology to fabricate inlays, onlays, and crowns. This is core technique and is a baseline of knowledge for ALL students. Throughout the clinical facility students are given the opportunity to utilize $\mathrm{CAD} / \mathrm{CAM}$ technology to treat clinical cases utilizing both direct and indirect techniques. The direct technique is taking a digital impression directly from the patient' mouth. The indirect technique is taking a conventional impression and fabricate a cast using stone. The digital impression is then taken from the stone cast many students reach independent competence in utilization of the technology and others, with minimal additional training will be able to supplement their knowledge and incorporate the fabrication of implant crowns, implant abutments, bridgework veneers as they advance in their career ${ }^{[5-7]}$. Computer Aided Design, throughout the manufacturing and design industry as well as dentistry, is a field in constant change and growth ${ }^{[1-7]}$.

\section{Methods and Materials}

In Biogeneric Copy mode, the preparation of the tooth is very important. Ledges and steps and bevels in the preparation must be avoided. Preparation undercuts must be eliminated at the margins. Internal preparation undercuts are not read by the camera, creating an internal void which fills with cement. Alternatively, internal undercuts may be eliminated with restorative agents such as composite or glass ionomer cements.

To permit proper milling of the material and to avoid concentration of the forces in the final porcelain restoration, all preparation angles should be rounded and smooth. The tooth is prepared with smooth surfaces and tapering walls to permit proper draw of the restoration and ease of digital impression taking. The occlusal surface reduction is critical. Proposed final thickness of the porcelain must be evaluated throughout the entire virtual restoration with special attention to the central fossa taking into account ideal depth and width. This is critical as final porcelain/composite restorations are subject to premature fracture during try-in phase and after cementation. The proximal box follows the similar ideal guidelines ${ }^{[2-4]}$. Using the software, the tooth number and the type of restoration, material selection (ceramic, polymer ceramic, or composite) is indicated and saved. Biogeneric Copy Mode [Figure 1,2] is intended for use in the design of inlay and onlay restorations.

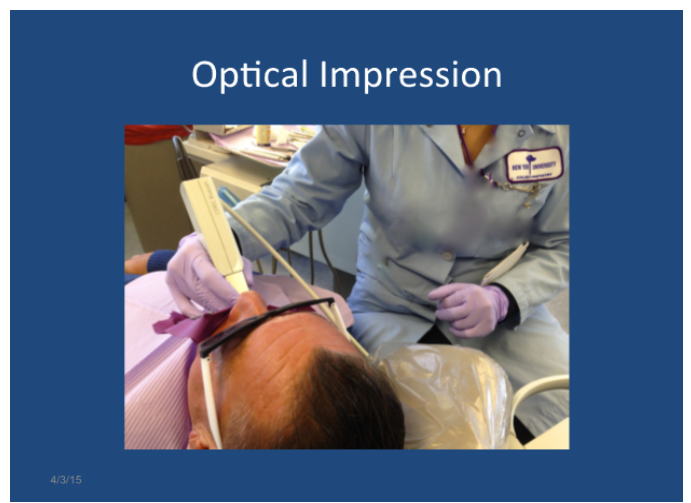

Figure 1: Biogeneric Copy- Imaging while utilizing rubber dam isolation

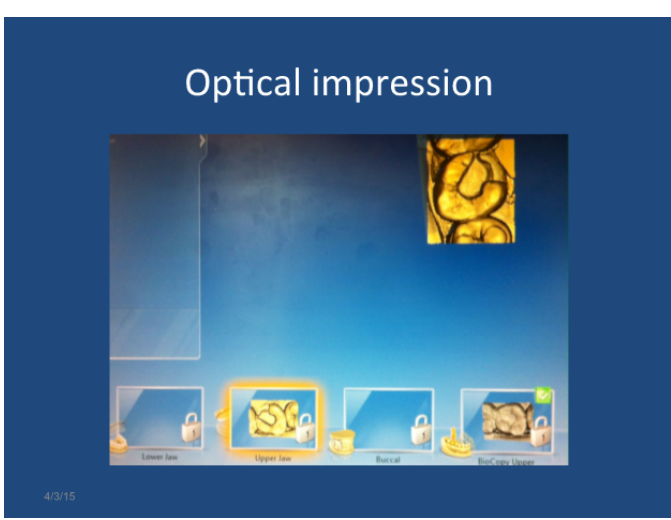

Figure 2: Biogeneric Copy

Isolation with rubber dam is necessary [Figure 3] to prevent saliva contamination. A digital impression of the intact unprepared tooth (even if it has a large failing restoration as long as the anatomy is desirable), including the mesial and distal structure of the neighboring teeth is taken with the camera on the acquisition unit. A second digital impression of the prepared tooth with the same path of insertion is taken. The software will copy and paste the occlusal part of the unprepared tooth, over the prepared tooth. If a tooth has a broken cusp the software will allow the ideal occlusal anatomy available in the library of information within the software to replace that cusp. The final design of the restoration will need little, if any, adjustment ${ }^{[2,3]}$.

Biogeneric Individual Mode [Figure 4] is utilized for the computer assisted design of full coronal restorations such as crowns. A digital impression is taken of the entire quadrant, including the prepared tooth. This is followed by a digital impression of the opposing quadrant, and a digital impression of the buccal view, and the upper and lower teeth in maximum inter-cuspation. The software is utilized to help design a three dimensional virtual model of the prepared tooth that is virtually trimmed and the restoration is designed, viewed in a buccal, lingual, mesial and distal aspect of the tooth. The adjacent teeth are analyzed and the software creates a proposal for a restoration with an ideal intercuspal relationship and anatomy. The dentist is able to modify contacts and contours of the restoration, as well as the occlusal surface anatomy and topography ${ }^{[2,3]}$.

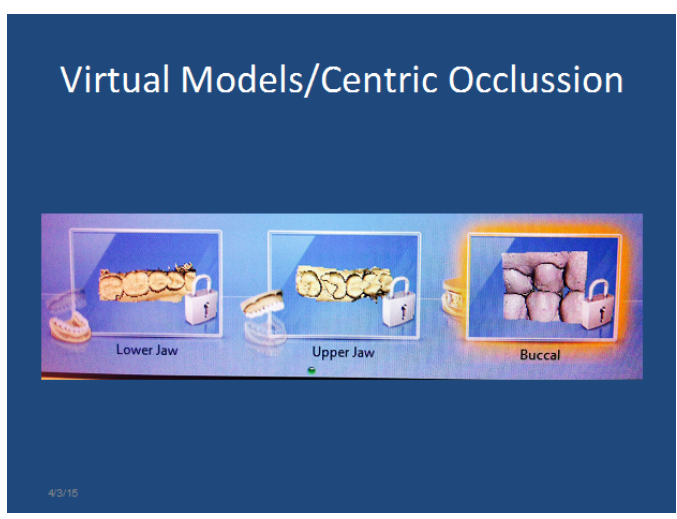

Figure 3: Biogeneric Individual 


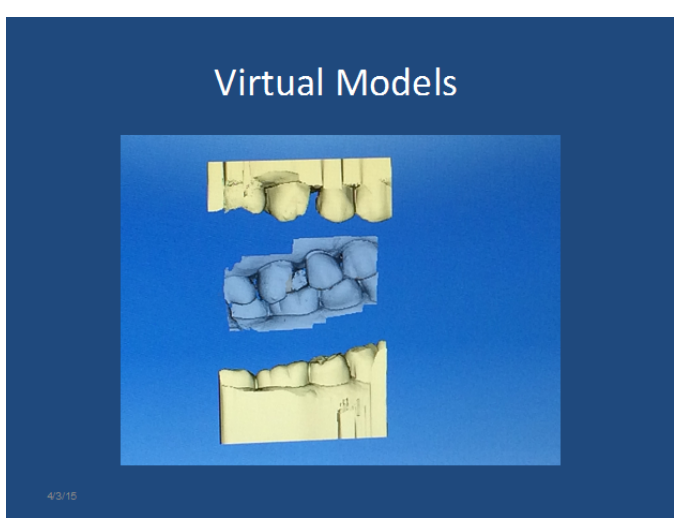

Figure 4: Biogeneric Individual

Once the operator is satisfied with the final design, the images are electronically sent to the milling chamber, generally located within 100 feet from the acquisition unit. After determining the appropriate shade, Cerec has a wide variety of materials such as Glass Ceramic, Pressed Ceramic, Polymer Ceramic and Composite with shades shades. The block of the chosen tooth shade and material is placed in the milling chamber and the milling process begins (usually takes between 10-20 minutes to complete). Once the restoration is milled, the sprue site is polished off, the restoration is tried in the patient's mouth assure appropriate margins, contacts, contours and shape adjusted if necessary and bonded in place.

\section{Discussion}

CAD/CAM technology has been in use in the dental field since the early 1980's when it was only limited to inlays and onlays ${ }^{[8,9]}$. Implementing this technology at NYUCD enabled the students to become early adopters and familiar with $\mathrm{CAD} / \mathrm{CAM}$ technology. As the software continues to improve over time the capabilities require significantly greater training to assure students are ready to utilize them.

The most recent software integrates with Galileo software for implant abutment positioning, creation of surgical stents prior to implant placement and then the ability to fabricate custom and stock abutments, in addition to the needed crown restoration $^{[5-7]}$. The information for surgical stents and implant placement can be digitally sent to the oral surgeon and to the dental technician.
Conventional tooth colored restorations can be more precise fitting and cost effective when using CAD/CAM technology ${ }^{[6,7]}$. The cost of CAD/CAM machines is significant however, if the practice has a patient population that is accepting of these restorations, it will be cost effective because lab fees will be eliminated, as well as cost of impression materials, and instrumentation relating to conventional impressions. In addition chair time is decreased and laboratory time is expedited ${ }^{[4-7]}$.

\section{Conclusion}

NYUCD is teaching the most basic techniques for inlays, onlays, and single unit crowns to all 380 students in our class. It is clinically acceptable to fabricate inlays and onlays with Biogeneric Copy or Biogeneric Individual modes. When fabricating single crowns Biogeneric Individual is utilized because the capability exists to modify the contours of the restoration in all dimensions, occlusal and inter proximal contacts and contours, as well as buccal and lingual contours. Training a student to do Biogeneric Copy and Biogeneric Individual better prepares them for the issues they will encounter in practice and broaden their horizon in doing CAD/CAM dentistry ${ }^{[8]}$.

\section{References}

1. Beuer, F., Schweiger, J., Edelhoff, D. Digital Dentistry: an overview of recent developments for CAD/CAM generated restorations. (2008) British Dental Journal 204: 505-511.

2. Apltauer, J. J., Oen, K. T., De Bartolo, A., et al. Engage: Teaching Pre-Doctoral Students State Of The Art Dentistry Using Cad/Cam Technology. (2012) Inted2012 Proceedings 2209-2211.

3. Hershkowitz, D., Allen, K., Estafan, D. Using Digital Imaging To Provide Student Feedback: An Educational Use Of Cad/Cam Technology. (2012) ICERI2012 Proceedings 4489-4491.

4. Haurtunian, M., De Bartolo, A., Klaczany, G., et al. Do You Want Your Student Doctors To Excel? (2012) ICERI2012 Proceedings 30833086.

5. Roberts, M. Putting It Together: Integrating New Digital and Restorative Options. (2013) Inside Dentistry 9(3).

6. Harsono, M., Simon, J. F., Stein, J. M., et al. Evolution of Chairside CAD/CAM Dentistry Dentists. (2012) Inside Dentistry 8: (10).

7. Birnbaum, N. S., Aaronson, H. B. Digital Dental Impression Systems There have been a number of recent advancements and integration into everyday dental practice. (2011) Inside Dentistry 7: (2).

8. Cardenas, M. R., Hershkowitz, D., Culotta, D. T., et al. TE-004 Going Beyond the Student's Potential by Teaching Them Two Different Innovative Modes to Fabricate Tooth Colored Restorations Using CAD/ CAM Technology (2015).

9. Rekow, D. Computer-aided design and manufacturing in dentistry: a review of the state of the art. (1987) J Prosthet Dent 58(4): 512-516.
Ommega Online Publishers

Journal Title: Journal of Dentistry and Oral Care

Journal Short Name: J Dent \& Oral Care
ISSN: 2379-1705

E-mail: dentistry@ommegaonline.com

Website: www.ommegaonline.org 